\title{
APPLICATION OF CHAOS DEGREE TO SOME DYNAMICAL SYSTEMS
}

\author{
$\dagger$ Kei Inoue, †Masanori Ohya and $\ddagger$ Keiko Sa to \\ $\dagger$ Department of Information Sciences, \\ Science University of Tokyo, \\ Noda City, Chiba 278-8510, Japan. \\ $\ddagger$ Department of Control and Computer Engineering, \\ Numazu College of Technology, \\ Numazu City, Shizuoka 410-8501, Japan
}

\begin{abstract}
Chaos degree defined through two complexities in information dynamics is applied to some deterministic dynamical models. It is shown that this degree well describes the chaostic feature of the models.
\end{abstract}

\section{Introduction}

There exist several approaches in the study of chaotic behavior of dynamical systems using the concepts such as entropy, complexity, chaos, fractal, stochasticity [1], 3, 4, 5, 6, 13]. In 1991, one of the authors proposed Information Dynamics (ID for short) [10, 11] to treat such chaotic behavior of systems altogether. ID is a synthesis of the dynamics of state change and the complexity of systems, and it is applied to several different fields such as quantum physics, fractal theory, quantum information and genetics [7].

A quantity measuring chaos in dynamical systems was defined by means of two complexities in ID, and it is called chaos degree. In particular, among several chaos degrees, an entropic chaos degree was introduced in [12, 8], and is applied to logistic map to study its chaotic behavior. This chaos degree 
has several merits compared with usual measures of chaos such as Lyapunov exponent.

In Section2, we review the complexity in ID and the chaos degree (CD for short). In Section 3, we remind the entropic chaos degree and the Lyapunov exponent (LE for short). In Section 4, the algorithm computing the entropic chaos degree is shown. In Section 5, we compute CD and LE for Bernoulli shift, Baker's transformation and Tinkerbell's map, then we discuss the merits of the entropic chaos degree.

\section{Complexity of Information Dynamics and Chaos Degree}

Information dynamics provides a frame to study the state change and the complexity associated with a dynamical system. We briefly explain the concept of the complexity of ID in a bit simplified version (see[1]).

Let $(\mathcal{A}, \mathfrak{S}, \alpha(G))$ be an input (or initial) system and $(\overline{\mathcal{A}}, \sqrt[\mathfrak{S}]{ }, \bar{\alpha}(\bar{G}))$ be an output (or final) system. Here $\mathcal{A}$ is the set of all objects to be observed and $\mathfrak{S}$ is the set of all means for measurement of $\mathcal{A}, \alpha(G)$ is a certain evolution of system. Often we have $\mathcal{A}=\overline{\mathcal{A}}, \mathfrak{S}=\overline{\mathfrak{S}}, \alpha=\bar{\alpha}$.

For instance, when $\mathcal{A}$ is the set $M(\Omega)$ of all measurable functions on a measurable space $(\Omega, \mathcal{F})$ and $\mathfrak{S}(\mathcal{A})$ is the set $P(\Omega)$ of all probability measures on $\Omega$, we have usual probability theory, by which the classical dynamical system is described. When $\mathcal{A}$ is the set $B(\mathcal{H})$ of all bounded linear operators on a Hilbert space $\mathcal{H}$ and $\mathfrak{S}(\mathcal{A})$ is the set $\mathfrak{S}(\mathcal{H})$ of all density operators on $\mathcal{H}$, we have a quantum dynamical system.

Once an input and an output systems are set, the situation of the input system is described by a state, an element of $\mathfrak{S}$, and the change of the state is expressed by a mapping from $\mathfrak{S}$ to $\overline{\mathfrak{S}}$, called a channel. The concept of channel is fundamental both in physics and mathematics [7]. Moreover, there exist two complexities in ID, which are axiomatically given as follows:

Let $\left(\mathcal{A}_{t}, \mathfrak{S}_{t}, \alpha^{t}\left(G^{t}\right)\right)$ be the total system of $(\mathcal{A}, \mathfrak{S}, \alpha)$ and $(\overline{\mathcal{A}}, \overline{\mathfrak{S}}, \bar{\alpha})$, and let $C(\varphi)$ be the complexity of a state $\varphi$ and $T\left(\varphi ; \Lambda^{*}\right)$ be the transmitted complexity associated with the state change $\varphi \rightarrow \Lambda^{*} \varphi$. These complexities $C$ and $T$ are the quantities satisfying the following conditions:

(i) For any $\varphi \in \mathfrak{S}$,

$$
C(\varphi) \geq 0, T\left(\varphi ; \Lambda^{*}\right) \geq 0
$$


(ii) For any orthogonal bijection $j: \operatorname{ex} \mathfrak{S} \rightarrow e x \mathfrak{S}$ ( the set of all extreme points in $\mathfrak{S}$ ),

$$
\begin{aligned}
C(j(\varphi)) & =C(\varphi), \\
T\left(j(\varphi) ; \Lambda^{*}\right) & =T\left(\varphi ; \Lambda^{*}\right) .
\end{aligned}
$$

(iii) For $\Phi \equiv \varphi \otimes \psi \in \mathfrak{S}_{t}$,

$$
C(\Phi)=C(\varphi)+C(\psi) .
$$

(iv) For any state $\varphi$ and a channel $\Lambda^{*}$,

$$
0 \leq T\left(\varphi ; \Lambda^{*}\right) \leq C(\varphi) .
$$

(v) For the identity map "id" from $\mathfrak{S}$ to $\mathfrak{S}$.

$$
T(\varphi ; i d)=C(\varphi) .
$$

When a state $\varphi$ changes to the state $\Lambda^{*} \varphi$, a chaos degree (CD) [11] w.r.t. $\varphi$ and $\Lambda^{*}$ is given by

$$
D\left(\varphi ; \Lambda^{*}\right)=C\left(\Lambda^{*} \varphi\right)-T\left(\varphi ; \Lambda^{*}\right) .
$$

Using the above $\mathrm{CD}$, we observe chaos of a dynamical system as

$$
\begin{aligned}
& C D>0 \Longleftrightarrow \text { chaotic. } \\
& C D=0 \Longleftrightarrow \text { stable. }
\end{aligned}
$$

\section{Entropic Chaos Degree and Lyapunov Ex- ponent}

Chaos degree in ID was applied to a smooth map on $R$ and it is shown that this degree enables to describe the chaotic aspects of a logistic map as well [8, 12]. Here we briefly review the chaos degree defined through classical entropies. For an input state described by a probability distribution $p=\left(p_{i}\right)$ and the joint distribution $r=\left(r_{i, j}\right)$ between $p$ and the output state $\bar{p}=$ $\Lambda^{*} p=\left(\bar{p}_{i}\right)$ through a channel $\Lambda^{*}$, the Shannon entropy $S(p)=-\sum_{i} p_{i} \log p_{i}$ 
and the mutual entropy $I\left(p ; \Lambda^{*}\right)=\sum_{i, j} r_{i, j} \log \frac{r_{i, j}}{p_{i} \bar{p}_{j}}$ satisfy all conditions of the complexities $C$ and $T$, then the entropic chaos degree is defined by

$$
\begin{aligned}
D\left(p ; \Lambda^{*}\right) & \equiv C\left(\Lambda^{*} p\right)-T\left(p ; \Lambda^{*}\right) \\
& \equiv S(\bar{p})-I\left(p ; \Lambda^{*}\right),
\end{aligned}
$$

This entropic chaos degree is nothing but the conditional entropy of aposteriori state w.r.t. the channel $\Lambda^{*}$. The characteristic point of the entropic chaos degree is easy to get the probability distribution of the orbit for a deterministic dynamics, which is discussed in the next section.

Lyapunov exponent (LE) is used to study chaotic behavior of a deterministic dynamics. The Lyapunov exponent $\lambda(f)$ for a smooth map $f$ on $\mathbf{R}$ is defined by [2]:

$$
\lambda(f)=\lim _{n \rightarrow \infty} \lambda_{n}(f) \lambda_{n}(f)=\frac{1}{n} \sum_{k=1}^{n} \log \left|\frac{d f}{d x}\left(x^{(k)}\right)\right|,
$$

where $x^{(n)}=f\left(x^{(n-1)}\right)$ for any $n \in \mathbf{N}$.

For a smooth map $f=\left(f_{1}, \cdots, f_{m}\right)$ on $\mathbf{R}^{\mathbf{m}}$, the vector version of $\mathrm{LE}$ is defined as follows: Let $x^{0}$ be an initial point of $\mathbf{R}^{\mathbf{m}}$ and $x^{(n)}=f\left(x^{(n-1)}\right)$ for any $n \in \mathbf{N}$. After $n$ times iterations of $f$ to $x^{0}$, the Jacobi matrix $J_{n}\left(x^{0}\right)$ of $x^{(n)}=\left(x_{1}^{(n)}, \cdots, x_{m}^{(n)}\right)$ w.r.t. $x^{(0)}$ is

$$
J_{n}\left(x^{(0)}\right)=D f^{n}\left(x^{(0)}\right)=\left[\begin{array}{lll}
\frac{\partial f_{1}^{(n)}}{\partial x_{1}}\left(x^{(0)}\right) & \cdots & \frac{\partial f_{1}^{(n)}}{\partial x_{m}}\left(x^{(0)}\right) \\
\cdots & \cdots & \cdots \\
\frac{\partial f_{m}^{(n)}}{\partial x_{1}}\left(x^{(0)}\right) & \cdots & \frac{\partial f_{m}^{(n)}}{\partial x_{m}}\left(x^{(0)}\right)
\end{array}\right],
$$

Then the Lyapunov exponent $\lambda(f)$ of $x^{(0)}$ is defined by

$$
\lambda(f)=\log \bar{\mu}_{1}, \bar{\mu}_{k}=\lim _{n \rightarrow \infty}\left(\mu_{k}^{n}\right)^{\frac{1}{n}}(k=1,2, \cdots, m) .
$$

Here $\mu_{k}^{n}$ is the $k$ th largest square root of the eigenvalues of the matrix $J_{n}\left(x^{(0)}\right) J_{n}\left(x^{(0)}\right)^{T}$.

An orbit of the dynamical system described by $f$ is said to be chaotic when the exponent $\lambda(f)$ is positive, and to be stable when the exponent is negative. The positive exponent means that the orbit is very sensitive to the initial value, so that it describes a chaotic behavior. Lyapunov exponent is difficult to compute for some models (e.g., Tinkerbell map) and its negative value is not clearly explained. 


\section{Algorithm for Computation of Entropic Chaos Degree}

It is proved [9] that if a piecewise monotone mapping $f$ from $[a, b]^{m}$ to $[a, b]^{m}$ has non-positive Schwarzian derivatives and does not have a stable and periodic orbit, then there exists an ergodic measure $\mu$ on the Borel set $\mathfrak{S}$ of $[a, b]^{m}$, absolutely continuous w.r.t. the Lebesgue measure.

Take a finite partition $\left\{A_{k}\right\}$ of $I=[a, b]^{m}$ such as

$$
I=\bigcup_{k} A_{k} \quad\left(A_{i} \bigcap A_{j}=\emptyset, i \neq j\right) .
$$

Let $|S|$ be the number of the elements in a set $S$. Suppose that $n$ is sufficiently large natural number and $m$ is a fixed natural number. Let $p^{(n)} \equiv\left(p_{i}^{(n)}\right)$ be the probability distribution of the orbit up to $n$-th step, that is, how many $x^{(k)}(k=m+1, \cdots, m+n)$ are in $A_{i}$ :

$$
p_{i}^{(n)} \equiv \frac{\left|\left\{k \in \mathbf{N} ; x^{(k)} \in A_{i}, m<k \leq m+n\right\}\right|}{n}
$$

It is shown that the $n \rightarrow \infty$ limit of $p_{i}^{(n)}$ exists and equals to $\mu\left(A_{i}\right)$. The channel $\Lambda^{*}$ is a map given by $p^{(n+1)}=\Lambda^{*} p^{(n)}$. Further, the joint distribution $r^{(n, n+1)}=\left(r_{i, j}^{(n, n+1)}\right)$ for a sufficient large $n$ is approximated as

$$
r_{i, j}^{(n, n+1)}=\frac{\left|\left\{k \in \mathbf{N} ;\left(x^{(k)}, f\left(x^{(k)}\right)\right) \in A_{i} \times A_{j}, m<k \leq m+n\right\}\right|}{n} .
$$

Then the entropic chaos degree is computed as

$$
\begin{aligned}
D\left(p^{(n)} ; \Lambda^{*}\right) & =S\left(p^{(n+1)}\right)-I\left(p^{(n)} ; \Lambda^{*}\right) \\
& =-\sum_{i} p_{i}^{(n+1)} \log p_{i}^{(n+1)}-\sum_{i, j} r_{i, j}^{(n, n+1)} \log \frac{r_{i, j}^{(n, n+1)}}{p_{i}^{(n)} p_{j}^{(n+1)}}
\end{aligned}
$$

\section{Entropic Chaos Degree for Some Determi- nistic Dynamical Models}

In this section, we study the chaotic behavior of several well-known deterministic maps by the entropic chaos degree. 


\section{$\langle 1\rangle$ Bernoulli shift}

Let $f$ a map from $[0,1]$ to itself such that

$$
f\left(x_{n}\right)= \begin{cases}2 a x^{(n)} & \left(0 \leq x^{(n)} \leq 0.5\right) \\ a\left(2 x^{(n)}-1\right) & \left(0.5<x^{(n)} \leq 1\right)\end{cases}
$$

where $x^{(n)} \in[0,1]$ and $0 \leq a \leq 1$.

Let us compute the Lyapunov exponent and the entropic chaos degree (ECD for short) for the above Bernoulli shift $f$.

The orbit of the equation (5.1) is shown in Fig. 1:

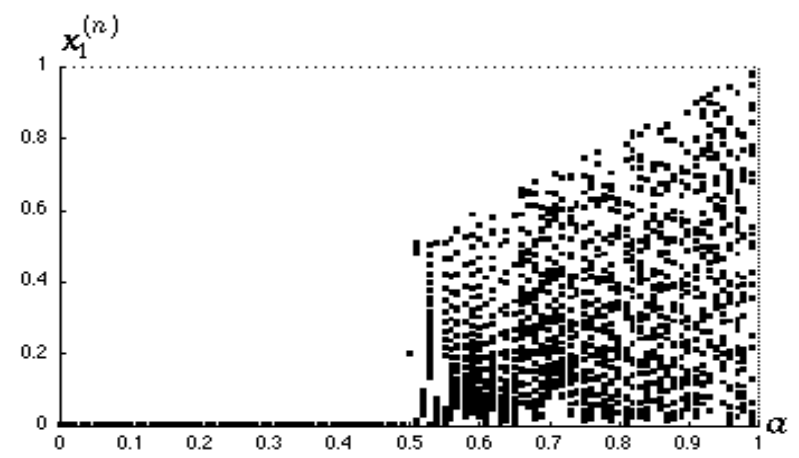

Fig1. The bifurcation diagram for Bernoulli shift

The Lyapunov exponent $\lambda_{n}(f)$ is $\log 2 a$ for the Bernoulli shift (Fig. 2).

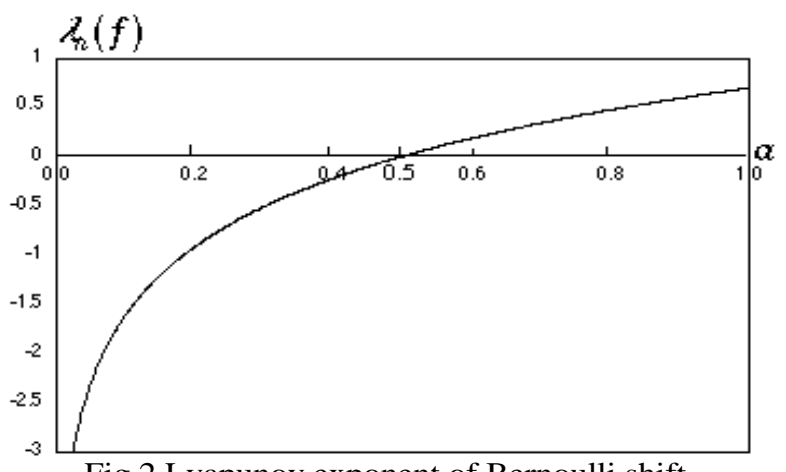

Fig.2 Lyapunov exponent of Bernoulli shift

On the other hand, the entropic chaos degree of the Bernoulli shift is shown in Fig. 3. 


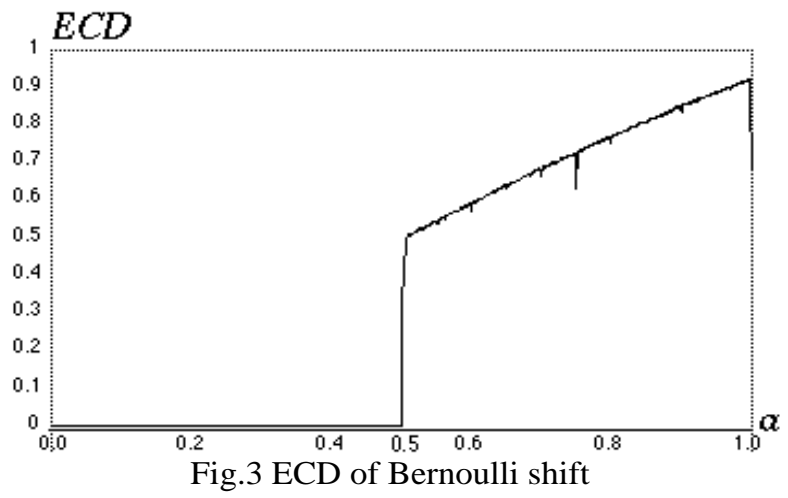

Here we took 740 different $a$ 's between 0 and 1 with

$$
\begin{aligned}
A_{i} & =\left[\frac{i}{2000}, \frac{i+1}{2000}\right] \quad(i=0, \cdots, 1999) \\
n & =100000
\end{aligned}
$$

\section{$\langle 2\rangle$ Baker's transformation}

We apply the chaos degree to a smooth map on $R^{2}$. Let us compute the Lyapunov exponent and the ECD for the following Baker's transformation $f_{a}$ :

$$
\begin{aligned}
f_{a}\left(x^{(n)}\right) & =f_{a}\left(x_{1}^{(n)}, x_{2}^{(n)}\right) \\
& =\left\{\begin{array}{ll}
\left(2 a x_{1}^{(n)} \frac{1}{2} a x_{2}^{(n)}\right) \\
\left(a\left(2 x_{1}^{(n)}-1\right), \frac{1}{2} a\left(x_{2}^{(n)}+1\right)\right) & \left(0 \leq x_{1}^{(n)} \leq 0.5\right) \\
0.5<x_{1}^{(n)} \leq 1
\end{array}\right)
\end{aligned}
$$

where $\left(x_{1}^{(n)}, x_{2}^{(n)}\right) \in[0,1] \times[0,1]$ and $0 \leq a \leq 1$. 
The orbit for each $a$ is shown in Fig. 4. $\sim 9$.

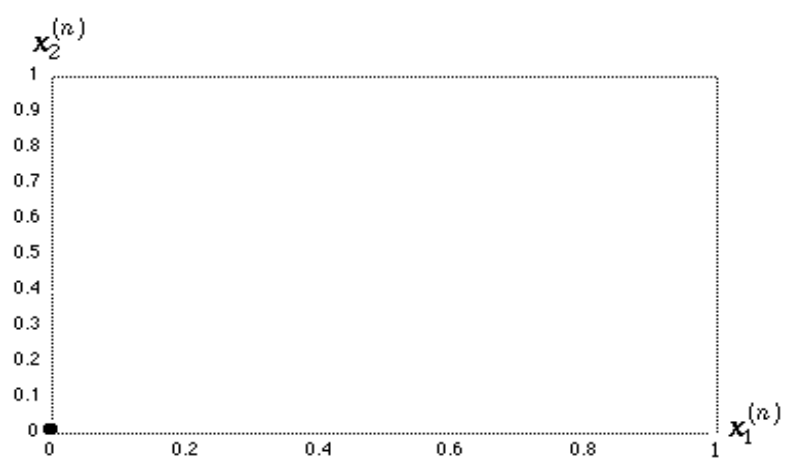

Fig.4 Orbit of $f_{a}$ w.r.t. $0 \leq a<0.5$

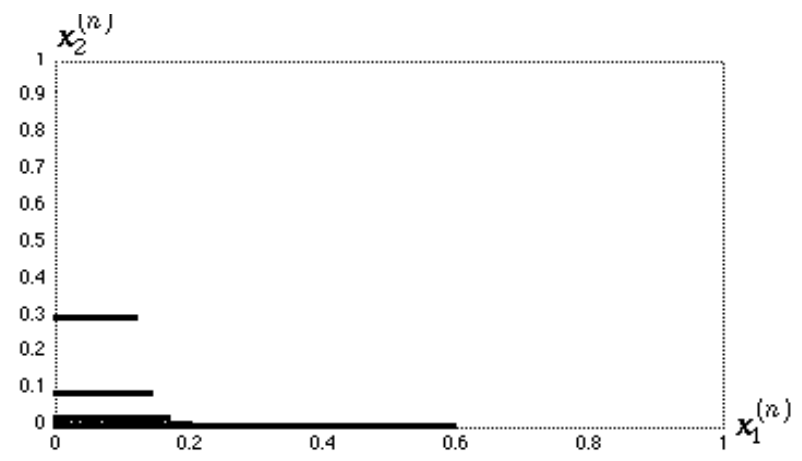

Fig.5 Orbit of $f_{a}$ w.r.t. $a=0.6$

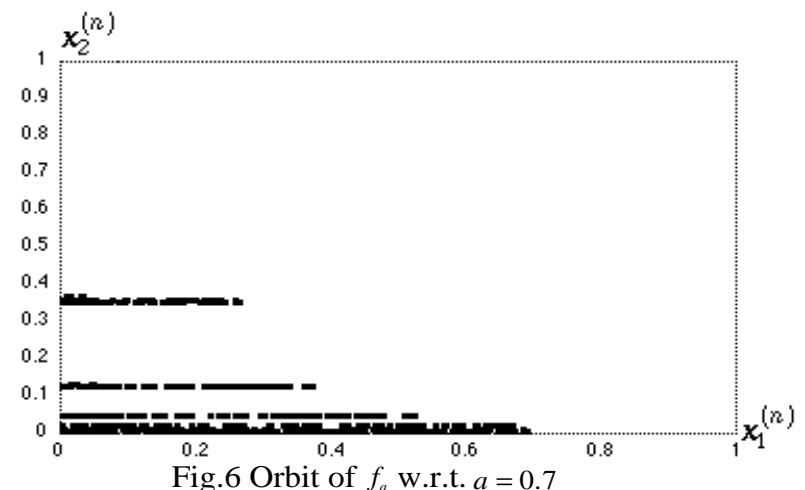




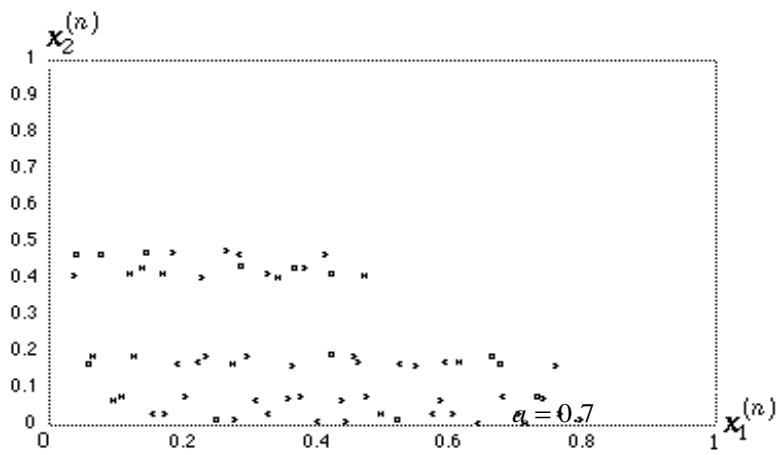

Fig.7 Orbit of $f_{a}$ w.r.t. $a=0.8$

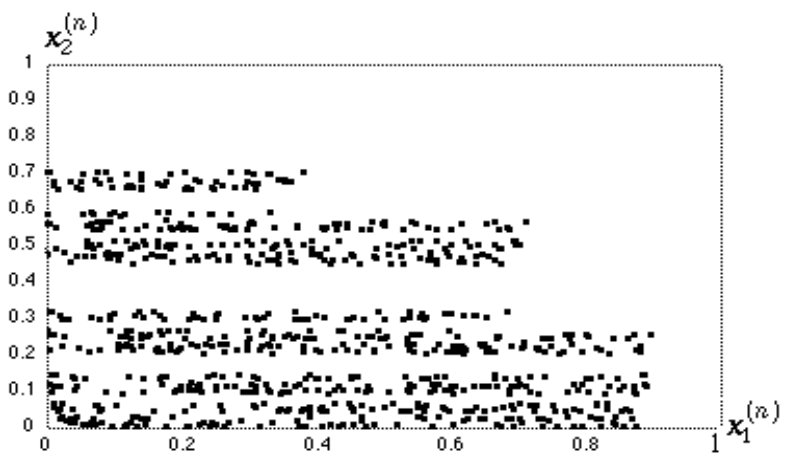

Fig. 8 Orbit of $f_{a}$ w.r.t. $a=0.9$

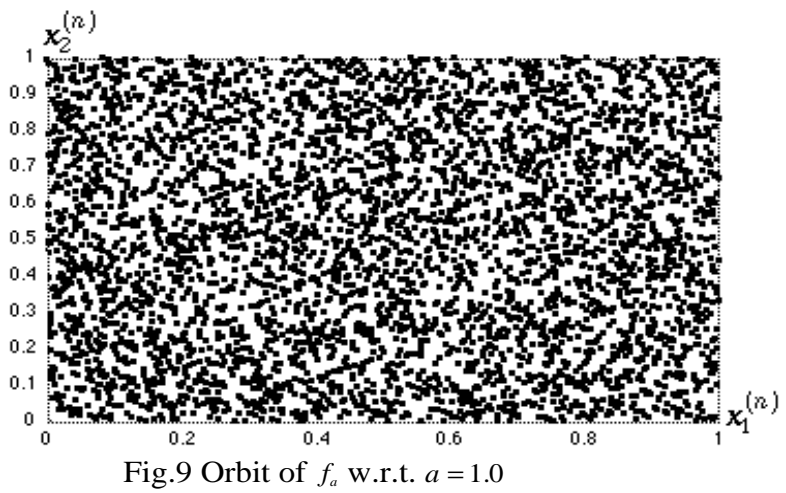

These figures show that the larger $a$ is, the more complicated the orbit is. The maximum Lyapunov exponent $\lambda_{n}^{1}(f)$ is $\log 2 a$ for Baker's transformation (Fig.10). 


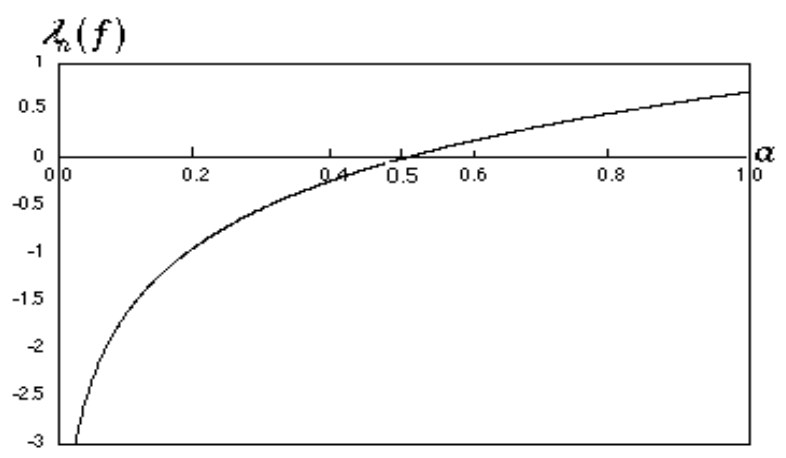

Fig. 10 Lyapunov exponent of Baker's transformation

On the other hand, the ECD of Baker's transformation is shown in Fig.11.

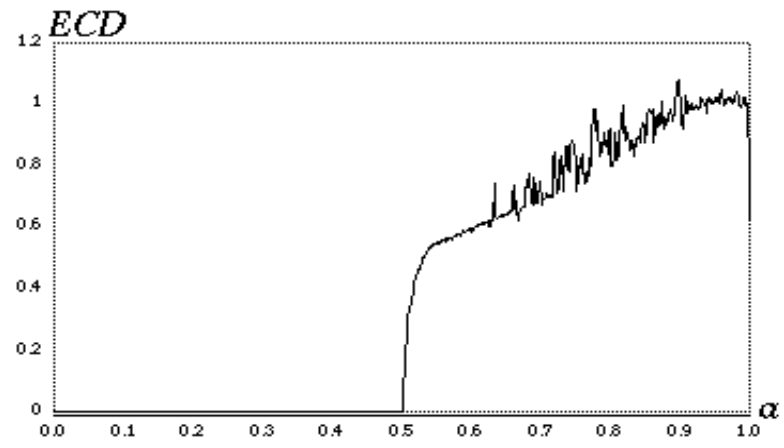

Fig. 11 ECD of Baker's transformation

Here we took 740 different $a$ 's between 0 and 1 with

$$
\begin{aligned}
A_{i, j} & =\left[\frac{i}{100}, \frac{i+1}{100}\right] \times\left[\frac{j}{100}, \frac{j+1}{100}\right] \quad(i, j=0, \cdots, 99) \\
n & =100000 .
\end{aligned}
$$

\section{$\langle 3\rangle$ Tinkerbell map}

Let us compute the CD for the following two type Tinkerbell maps $f_{a}$ and $f_{b}$ on $I=[-1.2,0.4] \times[-0.7,0.3]$. 


$$
\begin{aligned}
f_{a}\left(x^{(n)}\right) & =f_{a}\left(x_{1}^{(n)}, x_{2}^{(n)}\right) \\
& =\left(\left(x_{1}^{(n)}\right)^{2}-\left(x_{2}^{(n)}\right)^{2}+a x_{1}^{(n)}+c_{2} x_{2}^{(n)}, 2 x_{1}^{(n)} x_{2}^{(n)}+c_{3} x_{1}^{(n)}+c_{4} x_{2}^{(n)}\right) \\
f_{b}\left(x^{(n)}\right) & =f_{b}\left(x_{1}^{(n)}, x_{2}^{(n)}\right) \\
& =\left(\left(x_{1}^{(n)}\right)^{2}-\left(x_{2}^{(n)}\right)^{2}+c_{1} x_{1}^{(n)}+c_{2} x_{2}^{(n)}, 2 x_{1}^{(n)} x_{2}^{(n)}+b x_{1}^{(n)}+c_{4} x_{2}^{(n)}\right),
\end{aligned}
$$

where $\left(x_{1}^{(n)}, x_{2}^{(n)}\right) \in I,-0.4 \leq a \leq 0.9,1.9 \leq b \leq 2.9,\left(c_{1}, c_{2}, c_{3}, c_{4}\right)=$ $(-0.3,-0.6,2.0,0.5)$, and $\left(x_{1}^{(0)}, x_{2}^{(0)}\right)=(0.1,0.1)$.

Let us plot the points $\left(x_{1}^{(n)}, x_{2}^{(n)}\right)$ for 3000 different $n$ 's between 1001 and 4000.

In stable domain, the number of the points $\left(x_{1}^{(n)}, x_{2}^{(n)}\right)$ is finite because the point $\left(x_{1}^{(n)}, x_{2}^{(n)}\right)$ periodically appears in time $n$. Fig.12 and Fig.13 are examples of the orbits of $f_{a}$ and $f_{b}$ in a stable domain.

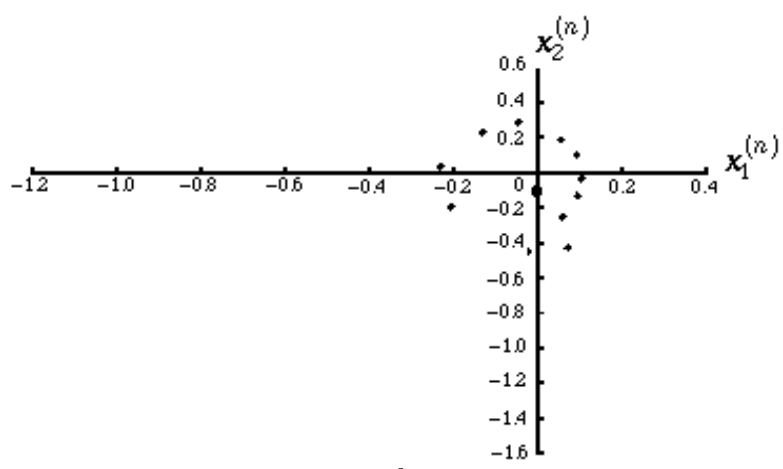

Fig.12 Orbit of $f_{a}$ w.r.t. $a=0.243$ 


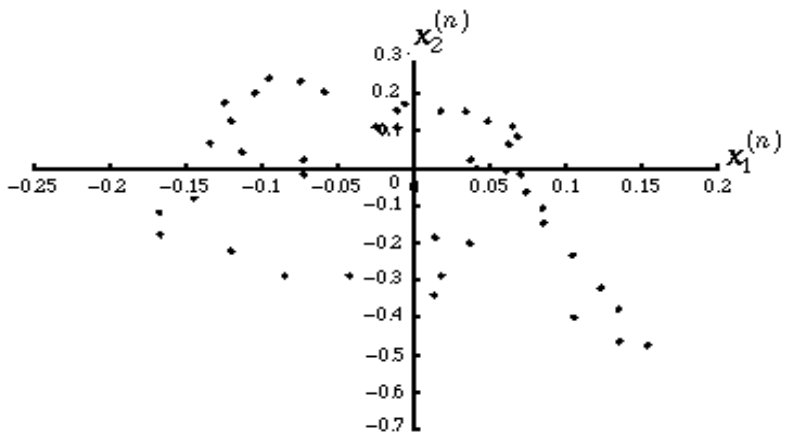

Fig. 13 Orbit of $f_{b}$ w.r.t. $b=2.65$

On the other hand, the point $\left(x_{1}^{(n)}, x_{2}^{(n)}\right)$ take random value in time $n$ in chaotic domain. Fig.514 and Fig.15 are examples of the orbits of $f_{a}$ and $f_{b}$ in a chaotic domain.

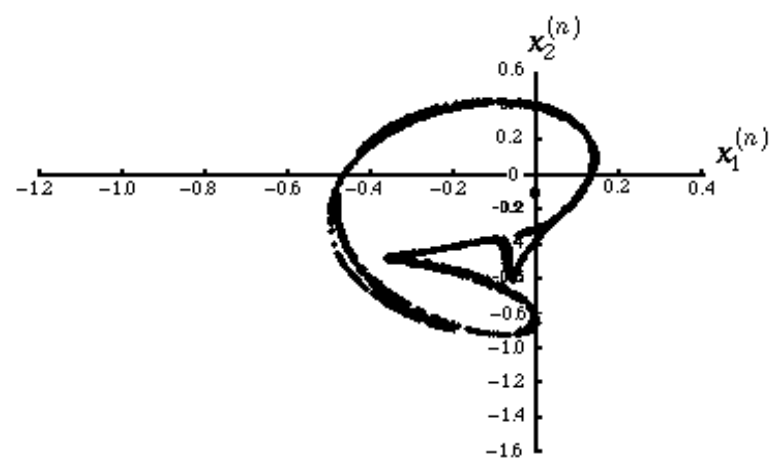

Fig.14 Orbit of $f_{a}$ w.r.t. $a=0.670$

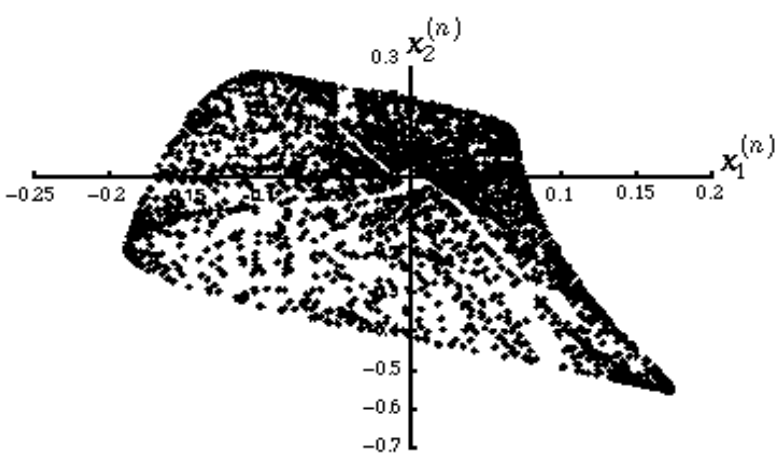

Fig.15 Orbit of $f_{b}$ w.r.t. $b=2.8$

The ECD of Tinkerbell map $f_{a}$ and $f_{b}$ are shown in Fig. 16 and Fig. 17. 


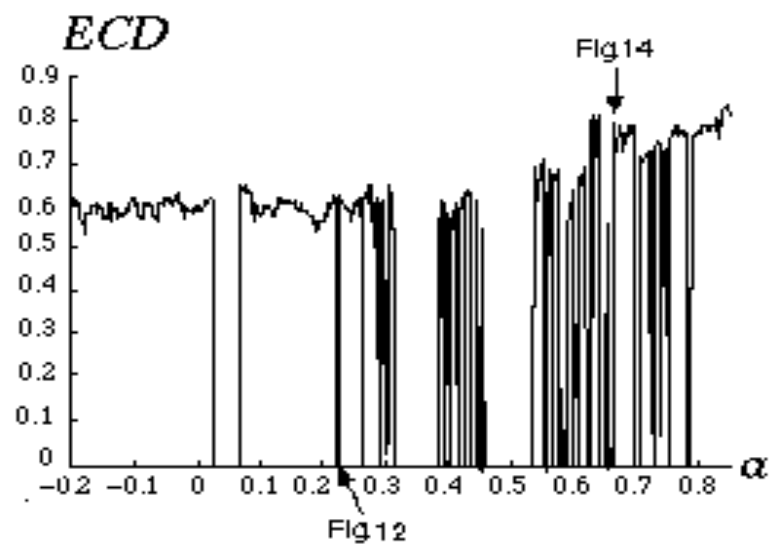

Fig. 16 ECD for Tinkerbell map $f_{a}$

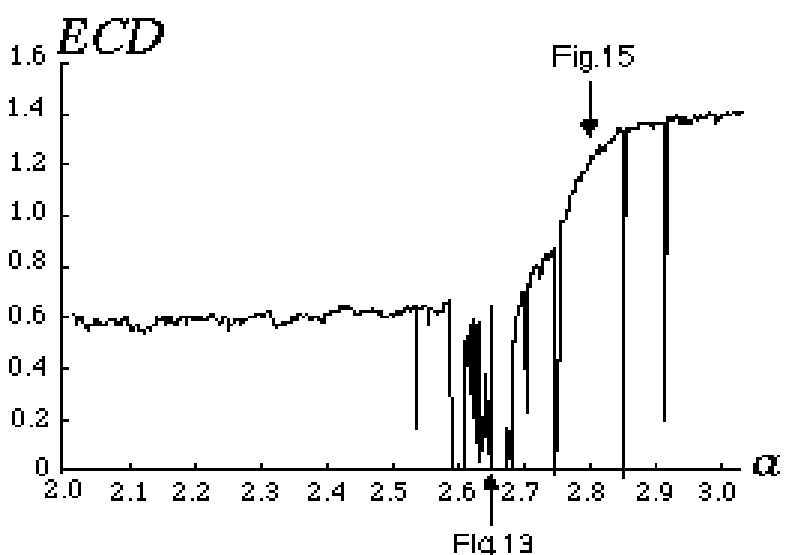

Fig.17 ECD for Tinkerbell map $f_{b}$

Here we took 740 different $a$ 's between -1.2 and 0.9 and 740 different $b$ 's between 1.9 and 2.9 with

$$
\begin{aligned}
A_{i, j}= & {\left[\frac{i}{100}, \frac{i+1}{100}\right] \times\left[\frac{j}{100}, \frac{j+1}{100}\right] } \\
& (i=-120,-119, \cdots,-1,0,1, \cdots, 38,39) \\
& (j=-70,-69, \cdots,-1,0,1, \cdots, 28,29) \\
n= & 100000 .
\end{aligned}
$$




\section{Conclusion}

From our results, when the orbits for Bernoulli shift, Baker's transformation or Tinkerbell map are chaotic, both Lyapunov exponent and chaos degree are positive. However, our chaos degree can resolve some inconvenient properties of the Lyapunov exponent in the following senses:

(1) Lyapunov exponent takes negative and sometimes $-\infty$. For instance, although the exponent of Bernoulli shift and Baker's transformation can not be defined for $a=0$, the ECD is always positive and defined for any $a \geq 0$.

(2) It is difficult to compute the Lyapunov exponent for the Tinkerbell maps $f_{a}$ and $f_{b}$ because it is difficult to compute $f_{a}^{n}$ and $f_{b}^{n}$ for large $n$. On the other hand, the ECD of $f_{a}$ and $f_{b}$ are easily computed.

(3) Generally, the algorithm for CD is much easier than that for Lyapunov exponent.

\section{References}

[1] Akashi, S., The asymptotic behavior of $\varepsilon$-entropy of a compact positive operator, J.Math.Anal.Appl., 153, 250, 1990.

[2] Alligood, K.T., Sauer, T.D., and Yorke, J.A., Chaos-An Introduction to Dynamical Systems-, Textbooks in Mathematical Sciences, Springer, 1996)

[3] Alicki, R., Quantum geometry of noncommutative Bernoulli shifts, Banach Center Publications, Mathematics Subject Classification 46L87, 1991.

[4] Bennatti, F., Deterministic Chaos in Infinite Quantum Systems, Springer, 1993.

[5] Devaney, R.L., An Introduction to Chaotic dynamical Systems, Benjamin, 1986.

[6] Hasegawa, H., Dynamical formulation of quantum level ststistics, Open Systems and Information dynamics, 4, 350, 1997. 
[7] Ingarden, R.S., Kossakowski, A. and Ohya, M., Information Dynamics and Open Systems, Kluwer Academic Publishers, 1997.

[8] Kosaka, M., and Ohya, M., A study of chaotic phenomena by information dynamics (in Japanese), IEICE, J80-A, No.12, 2138, 1997.

[9] Misiurewicz, M., Absolutely continuous measutres for certain maps of interval, Publ. Math. IHES, Vol.53, 17, 1981.

[10] Ohya, M., Information dynamics and its applications to optical communication processes, Lecture Note in Physics, 378, 81, 1991.

[11] Ohya, M., Complexity and fractal dimensions for quantum states, Open Systems and Information Dynamics, 4, 141, 1997.

[12] Ohya, M., Complexities and their applications to characterization of chaos, International Journal of Theoretical Physics, 37, No.1, 495, 1998.

[13] Toda, M., Crisis in chaotic scattering of a highly excited van der Waals complex, Physical Review Letters, 74, No.14, 2970, 1995. 